


\section{Surfactants \\ in Consumer Products}

Theory, Technology and Application

Edited by J. Falbe

With 260 Figures and 122 Tables

Springer-Verlag Berlin Heidelberg NewYork London Paris Tokyo 
Library of Congress Catalog Card Number.

Surfactants in consumer products. Includes bibliographies. 1. Surface active agents. I. Falbe, Jürgen. TP994.S876 $1986 \quad 668^{\prime} .1 \quad 86-24884$

ISBN-13:978-3-642-71547-1 (U.S.)

This work is subject to copyright. All rights are reserved, whether the whole or part of the material is concerned, specifically those of translation, reprinting, re-use of illustrations, broadcasting, reproduction by photocopying machine or similar means, and storage in data banks. Under $\S 54$ of the German Copyright Law, where copies are made for other than private use, a fee is payable to "Verwertungsgesellschaft Wort", Munich.

(C) by Springer-Verlag Heidelberg 1987

Sof tcover reprint of the hardcover 1st edition 1987

The use of registered names, trademarks, etc. in this publication does not imply, even in the absence of a specific statement, that such names are exempt from the relevant protective laws and regulations and therefore free for general use. 


\section{List of Contributors}

\section{Editor}

Prof. Dr. J. Falbe, Henkel KGaA, D-4000 Düsseldorf

\section{Contributors}

Dr. W. Adler, Henkel KGaA, D-4000 Düsseldorf

Dr. F. Bartnik, Henkel KGaA, D-4000 Düsseldorf

Dr. P. Berth, Henkel KGaA, D-4000 Düsseldorf

Dr. M. Biermann, Henkel KGaA, D-4000 Düsseldorf

D. Coons, Clorox Company, Pleasanton CA 94 566, USA

Dr. M. Dankowski, Degussa AG, D-6000 Frankfurt

Dr. M. Diehl, Degussa AG, D-6000 Frankfurt

Prof. Dr. J. Falbe, Henkel KGaA, D-4000 Düsseldorf

Dr. H. P. Fiedler, Hans Schwarzkopf GmbH, D-2000 Hamburg

Dr. P. Gerike, Henkel KGaA, D-4000 Düsseldorf

Dr. H. J. Heitland, Henkel KGaA, D-4000 Düsseldorf

Dr. G. Jakobi, Henkel KGaA, D-4000 Düsseldorf

Dr. P. Krings, Henkel KGaA, D-4000 Düsseldorf

Dr. K. Künstler, Henkel KGaA, D-4000 Düsseldorf

Dr. P. Kuzel, Degussa AG, D-6000 Frankfurt

Dr. F. Lange, Henkel KGaA, D-4000 Düsseldorf

S. N. Lewis, Clorox Company, Pleasanton CA 94 566, USA

Dr. H. Marsen, Thompson Siegel GmbH, D-4000 Düsseldorf

Prof. Dr. H. Offermanns, Degussa AG, D-6000 Frankfurt

Dr. R. Piorr, Henkel KGaA, D-4000 Düsseldorf

Dr. U. Ploog, Henkel KGaA, D-4000 Düsseldorf

Dr. H. Rutzen, Henkel KGaA, D-4000 Düsseldorf

Dr. F. Schambil, Henkel KGaA, D-4000 Düsseldorf

Dr. J. Schindler, Henkel KGaA, D-4000 Düsseldorf

Dr. E. Schmadel, Henkel KGaA, D-4000 Düsseldorf

Dr. R. Schmid, Henkel KGaA, D-4000 Düsseldorf

Dr. G. Schwarz, Henkel KGaA, D-4000 Düsseldorf

Dr. M. J. Schwuger, Henkel KGaA, D-4000 Düsseldorf

Dr. E. Sung, Henkel KGaA, D-4000 Düsseldorf

G. Thor, Henkel KGaA, D-4000 Düsseldorf

Dr. U. Trabitzsch, Henkel KGaA, D-4000 Düsseldorf

Dr. W. Umbach, Henkel KGaA, D-4000 Düsseldorf

Dr. E. Vaeth, Henkel KGaA, D-4000 Düsseldorf

Dr. H. Verbeek, Henkel KGaA, D-4000 Düsseldorf 


\section{Preface}

In todays market, custom formulated surfactants are offered for a wide range of applications. The need for surfactants in detergents, cleaning agents, cosmetics \& toiletries is second only to an expanding demand in industrial applications. But even within the non-industrial areas the demands have undergone significant changes in recent years. For example, washing and cleaning temperatures have substantially decreased with increased energy conservation attitudes, and more stringent regulatory requirements in the area of ecology and toxicology are leading to new product profiles. New manufacturing technologies and an increased utilization of natural raw materials also factor into this continuing evolution.

These changes and trends have been described in numerous publications. However, a summary and survey of these developments is currently missing. The book presented here

\section{"Surfactants in Consumer Products"}

is intended to close this gap.

The editor and authors dedicate this work to

\section{Dr. Dr. h.c. Konrad Henkel}

on the occasion of his 70 th birthday.

Dr. Henkel, himself a scientist and industrialist, contributed significantly to developments in the surfactant field. In the nineteen-fifties, he initiated the change from soap based detergents to synthetic detergents within Henkel. At the same time, dishwashing detergents utilizing various synthetic surfactants were also developed, and became the basis for modern manual and mechanical dishwashing.

Particularly noteworthy are Dr. Henkel's achievements in the promotion of the chemistry of fats - a continuously growing source of surfactant raw materials - and his initiatives in the systematic development of phosphate substitutes. In the nineteen-seventies, these latter endeavors were successfully crowned by the development of the ion exchanger, sodium aluminum silicate (zeolite A), which is now utilized world-wide.

The editor offers his appreciation to the many contributing authors from Henkel KGaA (Federal Republic of Germany), Clorox (USA), Degussa AG (Federal Republic of Germany) and Hans Schwarzkopf GmbH (Federal Republic of Germany), who made this book possible. I thank the Lion Corporation (Japan) for many valuable comments and contributions on several topics in this manuscript. 
The editor also owes special thanks to Dr. G. Jakobi (Henkel KGaA) for his thorough editorial work on the manuscripts, P. Leenders, Dr. M. Virnig and Dr. H.K. Maier (Henkel Corporation, Minneapolis) for their efforts in translating this work into English, Dr. M. Reinhardt and Dr. W. Stein (Henkel KGaA) for their critical reviews of the manuscripts and Dr. E. Köppelmann for coordinating support.

Thanks goes to the publishers for the cooperation in the planning and the smooth completion of this book.

Düsseldorf, October 1986

J. Falbe 


\section{Introduction}

Since the developement of the first synthetic surfactants in the 1930's, this product group has experienced extraordinary expansion in terms of the total volumes of product manufactured, the variety of chemical structures encompassed, and the number of areas of application.

The total world-wide output of surfactants in 1984 was about 14 million tons. The production of synthetic surfactants amounted to 5.3 million tons and that of soaps to 8.7 million tons. The areas of applications include industrial products and consumer products, such as, laundry detergents, dishwashing detergents, cleaning agents and cosmetic \& toiletries. In 1982, approximately $57 \%$ of all surfactants produced in the United States of America, Japan and Western Europe were employed in the manufacture of consumer products, thus strongly demonstrating the importance of this area of application. The world production of soaps, detergents and cleaning agents in 1984 was about 32 million tons, corresponding to a product value of more than 65 billion German Marks. The world cosmetics market had a total sales values in 1984 of more than 100 billion German Marks.

Within the last couple of years, the surfactant market has experienced increasingly frequent changes. This is due to variations in the raw material base (crude oil or natural oils/fats) and production technologies as well as changes in the requirements of the consumers and the legislators. This book, "Surfactant in Consumer Products", addresses these developments and the important areas of application.

In this book the physical-chemical principles of action of the surfactants, their production and their application in laundry detergents, dishwashing detergents and cleaning agents as well as in cosmetics \& toiletries are discussed. The technological aspects of application and formulation along with those of production and manufacturing processes are illustrated. Ecological and toxicological questions are probed in depth. Finally, important economic data concerning this branch of industry as well as an attempt to provide a perspective with regard to the future of the surfactant market round out the picture.

Our focus was not on a detailed rendering of all the historical developments in this field but rather on emphasizing the state-of-the-art of specific developments. While the aim of this book was not to completely record the total secondary literature the book will furnish the reader with a rather comprehensive compendium covering almost 1,400 literature citations. 
The book is primarily aimed at the experts in industry and in the universities. It will also, however, provide suggestions and references to those interested in general questions relating to surfactant chemistry, laundry detergents, cleaning agents, and cosmetics \& toiletries.

Due to the world-wide importance of the product groups addressed here, we strove to develop a truely international picture through cooperation of the staffs from Henkel KGaA (FRG), Degussa AG (FRG), and Hans Schwarzkopf GmbH (FRG) with those of The Clorox Company (USA), Lion Corporation (Japan) and Henkel Corporation (USA).

Prof. Dr. J. Falbe (Henkel KGaA)

Dr. S. N. Lewis (The Clorox Co.)

Prof. Dr. H. Offermanns (Degussa AG) 


\section{Contents}

Historical Review . . . . . . . . . . . . . . . . . . . . . 1

(Dr. H. Verbeek)

2 Structure and Application of Surfactants

(Dr. R. Piorr)

2.1 Surfactant Structure and Classification . . . . . . . . . . 5

2.2 Application of Surfactants . . . . . . . . . . . . . . 8

2.2.1 Consumer Products . . . . . . . . . . . . . . . . . . . . . 8

Detergents . . . . . . . . . . . . . 8

Dishwashing agents. . . . . . . . . . . . . . . 8

Cleaning agents . . . . . . . . . . . . 8

Personal products . . . . . . . . . . . . . . . 8

2.2.2 Industrial Applications . . . . . . . . . . . . . . . . . . . 9

Food products . . . . . . . . . . . . . . . . . . . . . 11

Pharmaceutical industry . . . . . . . . . . . . . . . . . . 11

Insecticides and herbicides . . . . . . . . . . . . . . . 11

Agriculture . . . . . . . . . . . . . . . . 12

Textiles and fibres . . . . . . . . . . . . . . . . . 12

Chemical industry . . . . . . . . . . . . . . . . . . . . 13

Plastics industry . . . . . . . . . . . . . . . . 14

Paints and lacquers . . . . . . . . . . . . . . . . . . 15

Cellulose and paper . . . . . . . . . . . . . . . . . . . . . 16

Leather and furs . . . . . . . . . . . . . . . . . . . 16

Photographic industry . . . . . . . . . . . . . . . . 17

Metal processing . . . . . . . . . . . . . . . . . . . . 18

Electroplating . . . . . . . . . . . . . . . . . . . . . 18

Adhesives . . . . . . . . . . . . . . . . . . . . . . . . . . . 19

Road construction and building materials . . . . . . . . . . 19

Firefighting . . . . . . . . . . . . . . . . 20

Mining and flotation . . . . . . . . . . . . . . . 20

Oilfield chemicals . . . . . . . . . . . . . . . 21

2.3 Literature ................... 22 
(Dr. M. Biermann, Dr. F. Lange, Dr. R. Piorr, Dr. U. Ploog,

Dr. H. Rutzen, Dr. J. Schindler, Dr. R. Schmid)

3.1 Raw Materials . . . . . . . . . . . . . . . 24

3.1.1 Fat Derived Chemical Raw Materials . . . . . . . . . . . . . 28

3.1.1.1 Fatty Acids . . . . . . . . . . . . . . . . . . . . . . . 30

3.1.1.2 Fatty Acid Methyl Esters. . . . . . . . . . . . . . . . . . . 34

3.1.1.3 Fatty Alcohols . . . . . . . . . . . . . . . . . . . . . . . 36

3.1.1.4 Fatty Amines . . . . . . . . . . . . . . . . . . . . . . . . 39

3.1.2 Petrochemical Raw Materials . . . . . . . . . . . . . . . . 39

3.1.2.1 Long-Chain Hydrocarbons . . . . . . . . . . . . . . . . . . 41

3.1.2.2 Short-Chain Hydrocarbons . . . . . . . . . . . . . . . . . . 43

3.1.2.3 Alkylene Oxides. . . . . . . . . . . . . . . . . . . . . . . 44

3.1.2.4 Olefins, Fatty- and Oxo-Alcohols - by Chain-Building Reactions . 45

3.1.2.5 Alkyl Aromatics . . . . . . . . . . . . . . . . . . . . . . 52

3.1.2.6 Amines . . . . . . . . . . . . . . . . . . . . 53

3.1.3 Fatty Chemical and Petrochemical Raw Materials -

Contrast or Supplement? . . . . . . . . . . . . . . . . . . . 53

3.2 Surfactants . . . . . . . . . . . . . . 54

3.2.1 Anionic Surfactants . . . . . . . . . . . . . . . . . . . . . 54

3.2.1.1 General Production Technologies . . . . . . . . . . . . . . . 54

3.2.1.2 Soaps . . . . . . . . . . . . . . . . . . . . . . . . . . 62

3.2.1.3 Alkylbenzene Sulfonates . . . . . . . . . . . . . . . . . . 63

3.2.1.4 Fatty Alcohol Sulfates . . . . . . . . . . . . . . . . . . . . 67

3.2.1.5 Alkyl Ether Sulfates . . . . . . . . . . . . . . . . . . . . . 67

3.2.1.6 Alkane Sulfonates . . . . . . . . . . . . . . . . . . . . . . 69

3.2.1.7 Olefin Sulfonates . . . . . . . . . . . . . . . . . . . . . . 71

3.2.1.8 $\alpha$-Sulfo Fatty Acid Esters. . . . . . . . . . . . . . . . . . . 75

3.2.1.9 Internal Sulfo Fatty Acid Esters . . . . . . . . . . . . . . . . 80

3.2.1.10 Fatty Acid Cyanamides (Amide Soaps) . . . . . . . . . . . . . 81

3.2.1.11 Sulfo Succinic Acid Alkyl Esters . . . . . . . . . . . . . . . 82

3.2.1.12 Acyl Oxyalkane Sulfonates (Fatty Acid Isethionates) . . . . . . . 83

3.2.1.13 Acylamino Alkane Sulfonates (Taurides) . . . . . . . . . . . . . 84

3.2.1.14 Ether Sulfonates . . . . . . . . . . . . . . . . . . . . . . 84

3.2.1.15 Ether Carboxylic Acids . . . . . . . . . . . . . . . . . . . 84

3.2.1.16 Sarcosinates . . . . . . . . . . . . . . . . . . . 85

3.2.1.17 Alkyl Phosphates and Alkyl Ether Phosphates . . . . . . . . . 85

3.2.2 Nonionic Surfactants . . . . . . . . . . . . . . . . . 86

3.2.2.1 Basic Properties . . . . . . . . . . . . . . . . . . . . . 86

3.2.2.2 Polyglycol Ethers . . . . . . . . . . . . . . . . . . . . . . 87

Fatty alcohol polyglycol ethers . . . . . . . . . . . . . . . 88

Alkylphenol polyglycol ethers . . . . . . . . . . . . . . . 90

End group-capped fatty alcohol polyglycol ethers . . . . . . . 91

Fatty acid polyglycol esters . . . . . . . . . . . . 92

Fatty acid alkanol amides, fatty acid alkanolamido

polyglycol ethers . . . . . . . . . . . . . . 93

Ethylene oxide/propylene oxide block polymers . . . . . . . . 96 
3.2.2.3 Polyol Surfactants . . . . . . . . . . . . . . . . . . . . . . 99

Glycerine fatty acid esters . . . . . . . . . . . . . . . . . . . 99

Sorbitan esters . . . . . . . . . . . . . . . . . . . . . . 99

Sugar derived surfactants (sugar esters, alkyl glycosides) . . . . 101

3.2.2.4 Amine Oxides . . . . . . . . . . . . . . . . . . . . . . 105

3.2.3 Cationic Surfactants . . . . . . . . . . . . . . . . . . . 106

3.2.3.1 Application Areas . . . . . . . . . . . . . . . . . . . . . . 106

3.2.3.2 Reaction Conditions . . . . . . . . . . . . . . . . . . . . . 107

Amine salts . . . . . . . . . . . . . . . . . . . . . . . 107

Quaternary ammonium compounds (quats) . . . . . . . . 107

3.2.3.3 Amines with one long Alkyl Group . . . . . . . . . . . . . . . 109

3.2.3.4 Amines with a long $\beta$-Hydroxyalkyl Group . . . . . . . . . . . . . 110

3.2.3.5 Amines with Several long Alkyl Groups . . . . . . . . . . . . . 111

3.2.3.6 Amines with Several long $\beta$-Hydroxyalkyl Groups . . . . . . . . 113

3.2.4 Amphoteric Surfactants . . . . . . . . . . . . . . . . . . . 114

3.2.4.1 Carboxy Amphoteric Surfactants . . . . . . . . . . . . . . . 115

Alkyl betaines . . . . . . . . . . . . . . . . . . 115

Other amphoterics . . . . . . . . . . . . . . 116

3.2.4.2 Sulfo Betaines . . . . . . . . . . . . . . . . . . . . . . . 117

3.2.4.3 Basic Properties . . . . . . . . . . . . . . . . . . . . . 118

3.2.5 Biosurfactants . . . . . . . . . . . . . . . . . . 118

3.2.5.1 Structure and Synthesis . . . . . . . . . . . . . . . . . . . 119

3.2.5.2 Applications . . . . . . . . . . . . . . . . . 122

3.3 Literature . . . . . . . . . . . . . . . . . . . . 124

4 Interfacial and Colloidal Properties . . . . . . . . . . . . . . . 133

(Dr. F. Schambil, Dr. M. J. Schwuger)

$4.1 \quad$ Solubility of Surfactants . . . . . . . . . . . . . . . . . 133

4.1.1 Krafft Point . . . . . . . . . . . . . . . . . . . . . . . 134

4.1.2 Cloud Point . . . . . . . . . . . . . . . . . . . . . . . . 137

4.2 Interfacial Activity and Micelle Formation . . . . . . . . . . . 139

4.2.1 Effect of Surfactant Constitution . . . . . . . . . . . . . . . 139

4.2.2 Properties of Surfactant Mixtures . . . . . . . . . . . . . . . 142

4.2.3 Oil Specificity of Interfacial Activity . . . . . . . . . . . . . . 146

4.2.4 Emulsions (HLB Values) . . . . . . . . . . . . . . . . . . . 149

4.2.5 Polymeric Surfactants . . . . . . . . . . . . . . . . . . 153

$4.3 \quad$ Spread and Adsorbed Monolayers . . . . . . . . . . . . . . 156

4.3.1 Properties of Monolayers . . . . . . . . . . . . . . . . 156

4.3.2 Gibbs' Film Elasticity and Marangoni Effect . . . . . . . . . . 158

4.3.3 Surface Shear Viscosity and Surface Dilatation Viscosity . . . . . 159

4.3.4 Foam Stability . . . . . . . . . . . . . . . . . . . . . 159

4.4 Surfactant Aggregates . . . . . . . . . . . . . . . . 163

4.4.1 Thermodynamics of Micelle Formation . . . . . . . . . . . . 163 
4.4.2 Kinetics of Micelle Formation . . . . . . . . . . . . . . . . 164

4.4.3 Properties of Micelles . . . . . . . . . . . . . . . . . . . . 166

4.4.4 Lyotropic Liquid Crystals . . . . . . . . . . . . . . . . . . 170

4.4.5 Microemulsions . . . . . . . . . . . . . . . . . . . . . 178

4.5 Adsorption on Solid Surfaces . . . . . . . . . . . . . . . . . 180

4.5.1 Nonpolar Solids . . . . . . . . . . . . . . . . . . . . . . 180

4.5.2 Polar Solids . . . . . . . . . . . . . . . . . . . . . . . . 182

4.6 Theory of Washing and Cleaning . . . . . . . . . . . . . . . 184

4.6.1 General Aspects . . . . . . . . . . . . . . . . . . . . . . . 184

4.6.2 Oily and Fatty Soil . . . . . . . . . . . . . . . . . . . . . 186

4.6.3 Effect of Surfactants on Solid Soil Particles . . . . . . . . . . . 188

4.6.4 Effect of Sequestrants and Ion Exchangers . . . . . . . . . . . 191

4.7 Literature . . . . . . . . . . . . . . . . 192

5 Performance in Detergents, Cleaning Agents

and Personal Care Products . . . . . . . . . . . . . . . . . . 197

5.1 Detergents . . . . . . . . . . . . . . . . 197

(D. Coons, Dr. M. Dankowski, Dr. M. Diehl, Dr. G. Jakobi,

Dr. P. Kuzel, Dr. E. Sung, Dr. U. Trabitzsch)

5.1.1 Application Aspects . . . . . . . . . . . . . . . . . . . . . 199

5.1.1.1 Partners in the Washing Process . . . . . . . . . . . . . . . . 199

Textiles . . . . . . . . . . . . . . . . . . . . . . . 199

Laundry soils . . . . . . . . . . . . . . . . . . 201

Water . . . . . . . . . . . . . . 204

Washing machines . . . . . . . . . . . . . 207

Detergents . . . . . . . . . . . . . . . 210

5.1.1.2 Wash Practices and Conditions in Various Countries/Continents . . 210

Textiles . . . . . . . . . . . . . . . 210

Wear and use time periods . . . . . . . . . . . . . . . 211

Washing machines and water hardness . . . . . . . . . . 211

Washing temperatures . . . . . . . . . . . . . . 211

Time of agitation. . . . . . . . . . . . . . . . . . 211

Detergent compositions . . . . . . . . . . . . . 213

5.1.1.3 Test Methods . . . . . . . . . . . . . . . . . . . . 215

Pure laboratory tests. . . . . . . . . . . . . . . . . 215

Artificially soiled test fabrics . . . . . . . . . . . 215

Detergency . . . . . . . . . . . . . . . . 217

Foaming . . . . . . . . . . . . . . . . . . 218

Fabric softeners . . . . . . . . . . . . . . . . . 219

Antiredeposition properties . . . . . . . . . . . . . 220

Incrustation . . . . . . . . . . . . . . . . . . . . . 220

Fabric damage . . . . . . . . . . . . . . . . . . . . . 220

Fabric color or dye damage . . . . . . . . . . . . . . . . 220

Brightening effects . . . . . . . . . . . . . 221

Physical characteristics . . . . . . . . . . . . . . . 221

Practical evaluation . . . . . . . . . . . . . . . . . 221

Consumer test . . . . . . . . . . . . . . . . . 223 
5.1.1.4 Surfactants - Structure/Effect Relationships . . . . . . . . . . . 223

Homologs . . . . . . . . . . . . . . . . . . . . . . . 224

Isomers . . . . . . . . . . . . . . . . . . . . . 229

Structures . . . . . . . . . . . . . . . . . . . . . 232

Sensitivity to water hardness . . . . . . . . . . . . 233

Counterion influence . . . . . . . . . . . . . . . 238

Adsorption and effect . . . . . . . . . . . . . . . . . . . 241

5.1.1.5 Textile Fiber and Detergency . . . . . . . . . . . . . . . . . 244

5.1.2 Detergent Ingredients . . . . . . . . . . . . . . . . . . . 247

5.1.2.1 Surfactants . . . . . . . . . . . . . . . . . . . . . . 247

Anionic surfactants . . . . . . . . . . . . . . . . . . . . 248

Nonionic surfactants . . . . . . . . . . . . . . 252

Cationic surfactants . . . . . . . . . . . . . . . . 254

Amphoteric surfactants . . . . . . . . . . . . 255

5.1.2.2 Builders . . . . . . . . . . . . . . . . . 255

Alkalis . . . . . . . . . . . . . . . . . . . . 256

Complexing agents . . . . . . . . . . . . . . . . . . 256

Ion exchangers. . . . . . . . . . . . . . . . . . . . . 262

5.1.2.3 Bleaching Systems . . . . . . . . . . . . . . . . . . . . . . 265

Peroxide or active oxygen bleach . . . . . . . . . . . 265

Hypochlorite bleaches . . . . . . . . . . . . . . 272

5.1.2.4 Auxiliary Agents . . . . . . . . . . . . . . . . . . . . . . 275

Enzymes . . . . . . . . . . . . . . . . . . . 275

Antiredeposition agents . . . . . . . . . . . . . . 277

Foaming stabilizers and regulators . . . . . . . . . . . 278

Fluorescent whitening agents or optical brighteners (FWA) . . 279

Corrosion inhibitors . . . . . . . . . . . . . . . . . . . 284

Perfumes . . . . . . . . . . . . . . . . . . . . . . . 284

Dyestuffs . . . . . . . . . . . . . . . . . . . . . . 284

Fillers and formulation aids . . . . . . . . . . . 285

5.1.3 Formulations of Household Detergents . . . . . . . . . . . . . 286

5.1.3.1 Heavy-Duty or All-Purpose Detergents . . . . . . . . . . . . . . . 286

Heavy-duty detergent powders . . . . . . . . . . . . . . . 286

Liquid heavy-duty detergents . . . . . . . . . . . . . 287

5.1.3.2 Specialty Detergents . . . . . . . . . . . . . . . . . . 289

Specialty detergent powders . . . . . . . . . . . . . . . . 289

Liquid specialty detergents. . . . . . . . . . . . . . . 291

5.1.3.3 Laundry Aids . . . . . . . . . . . . . . . . . . . . . . . . 291

Pretreatment aids . . . . . . . . . . . . . . . . . . . . 292

Laundry or water softeners . . . . . . . . . . . . . . . 292

Laundry presoaking products . . . . . . . . . . . . . . 293

Prewash soil and stain removers . . . . . . . . . . . 293

Boosters . . . . . . . . . . . . . . . . . . . . 293

Laundry bleaches . . . . . . . . . . . . . . . 293

Laundry boosters . . . . . . . . . . . . . . . . . . . 294

Aftertreatment aids . . . . . . . . . . . . . . . 295

Fabric softeners . . . . . . . . . . . . . . . . . 295

Starches and stiffeners . . . . . . . . . . . . . . . 296

Fabric formers . . . . . . . . . . . . . . . . . . . . . 297

Laundry dryer aids . . . . . . . . . . . . . . . . . . . . 297

5.1.4 Institutional Detergents . . . . . . . . . . . . . . . . . . . 297

5.1.4.1 Introduction . . . . . . . . . . . . . . . . . . . . . . . 297

5.1.4.2 Detergents and Auxiliary Agents . . . . . . . . . . . . . . . 299

Partially built detergents . . . . . . . . . . . . . . . . . 299 
Completely built detergents . . . . . . . . . . . . . . . 300

Detergents for the pre and main wash . . . . . . . . . . . . 301

Perborate containing detergents . . . . . . . . . . . . . . 301

Special detergents . . . . . . . . . . . . . . . . . . . . . 302

Wetting agents . . . . . . . . . . . . . 303

Bleaching agents . . . . . . . . . . . . . . . . 303

Disinfection additives . . . . . . . . . . . . . . . . . . 303

Souring agents . . . . . . . . . . . . . . . . . . . . . . 304

Fabric softeners . . . . . . . . . . . . . . . . . . . . . 304

Miscellaneous products . . . . . . . . . . . . . . . . 304

5.1.4.3 The Future Development of Institutional Detergents . . . . . . . 304

5.2 Dishwashing Detergents and Hard Surface Cleaners for Household and Institutional Purposes . . . . . . . . . . . . . . . . . . 306 (Dr. H. Heitland, Dr. H. Marsen)

5.2.1 Manual Dishwashing Agents . . . . . . . . . . . . . . . . 308

5.2.1.1 Application Aspects . . . . . . . . . . . . . . . . . . . . . 308

5.2.1.2 Test Methods and Determination of Use Value . . . . . . . . . . 309

5.2.1.3 Ingredients . . . . . . . . . . . . . . . . . . . . . . 314

Primary surfactants . . . . . . . . . . . . . . . . . . . . 314

Surfactant combinations . . . . . . . . . . . . . . . . 317

Secondary surfactants . . . . . . . . . . . . . . . . . . . 317

5.2.1.4 Formulations . . . . . . . . . . . . . . . . . . . . . . . . 318

5.2.1.5 Additional Properties . . . . . . . . . . . . . . . . . . . . . . 320

Skin compatibility . . . . . . . . . . . . . . . . . . 320

Oral ingestion . . . . . . . . . . . . . . . 320

5.2.2 Products for Mechanical Dishwashing . . . . . . . . . . . . . 321

5.2.2.1 Dishwashing Machines . . . . . . . . . . . . . . . . . . . . 321

5.2.2.2 Application Aspects . . . . . . . . . . . . . . . . . . . . . . . . 324

5.2.2.3 Test Methods and Determination of Use Value . . . . . . . . . 327

5.2.2.4 Formulations . . . . . . . . . . . . . . . . . . . . . . . . 329

Dishwashing detergents . . . . . . . . . . . . . . . . . . . 329

Rinse aids . . . . . . . . . . . . . . . . . . . . . . . 330

5.2.2.5 Practical Application of Dishwashing Detergents . . . . . . . . 332

5.2.3 All-Purpose Cleaners and Scouring Agents . . . . . . . . . . . 333

5.2.3.1 Application Aspects . . . . . . . . . . . . . . . . . . . . 333

5.2.3.2 Test Methods and Determination of Use Value . . . . . . . . . 335

5.2.3.3 Ingredients . . . . . . . . . . . . . . . . . . . . . . . . . 336

Surfactants . . . . . . . . . . . . . . . . . . 336

Complexing agents . . . . . . . . . . . . . . . . . . . . 339

Organic polymers . . . . . . . . . . . . . . . . . . . . . 339

Solvents . . . . . . . . . . . . . . . . . . . . . . . . 339

Microbicides. . . . . . . . . . . . . . . . . . . . . 339

Bleaching compounds . . . . . . . . . . . . . . . . . 340

Abrasives . . . . . . . . . . . . . . . . . 340

5.2.3.4 Formulations . . . . . . . . . . . . . . . . . . . . . . . . 340

All purpose cleaners . . . . . . . . . . . . . . . . . . 340

Scouring agents . . . . . . . . . . . . . . . . 342

5.2.4 Special Cleaners for Bathrooms and Toilets . . . . . . . . . . . 342

5.2.4.1 Floors and Walls . . . . . . . . . . . . . . . . . . . . . . . 342

5.2.4.2 Bathtubs . . . . . . . . . . . . . . . . . . 343

5.2.4.3 Toilets. . . . . . . . . . . . . . . . . . . . . . . . . . 343

Liquid acidic toilet cleaners . . . . . . . . . . . . . . 344 
Spreadable toilet cleaning powders . . . . . . . . . . . 344

Auto-active toilet bowl cleaners . . . . . . . . . . . . . . 344

5.2.5 Wash-and-Wax Products (One-Step Clean and Shine Floor Polishes) 346

Surfactants . . . . . . . . . . . . . . 346

Waxes ................. . 346

Polymers . . . . . . . . . . . . . . . . . . . 346

5.2.6 Carpet Cleaners . . . . . . . . . . . . . . . . . . . . . . . 347

5.2.6.1 Application Aspects . . . . . . . . . . . . . . . . . . . . . 347

5.2.6.2 Test Methods . . . . . . . . . . . . . . . . . . . . . . . . 348

5.2.6.3 Active Ingredients and Formulations . . . . . . . . . . . . . . . 348

Foam cleaners . . . . . . . . . . . . . . . . . . . . . . 348

Spray-extraction cleaners . . . . . . . . . . . . . . . . 348

Powder cleaners . . . . . . . . . . . . . . . . . . . . . 349

5.3 Cosmetics and Toiletries . . . . . . . . . . . . . . 350

(Dr. H. P. Fiedler, Dr. W. Umbach)

5.3.1 Definition of Cosmetic Products (Government Regulations) . . . . 352

5.3.2 Predominant Types of Cosmetic Products . . . . . . . . . . . 352

5.3.2.1 Solutions. . . . . . . . . . . . . . . . . . . . . . . . . 352

5.3.2.2 Colloidal Solutions (Gels) . . . . . . . . . . . . . . . . . . 352

5.3.2.3 Suspensions . . . . . . . . . . . . . . . . . . . . . . . . 354

5.3.2.4 Oils . . . . . . . . . . . . . . . . . . . . . . . . . 354

5.3.2.5 Ointments . . . . . . . . . . . . . . . . . . . . . 354

5.3.2.6 Emulsions . . . . . . . . . . . . . . . . . . 355

5.3.2.7 Creams . . . . . . . . . . . . . . . . . . . . 355

5.3.2.8 Soaps . . . . . . . . . . . . . . . . . . . . . . 355

5.3.2.9 Surfactant Solutions (Liquid Soaps) . . . . . . . . . . . . . . 355

5.3.2.10 Sticks . . . . . . . . . . . . . . . . . . . . . . 356

5.3.2.11 Powders . . . . . . . . . . . . . . . . . . . . . 356

5.3.2.12 Granulates . . . . . . . . . . . . . . . . . . . . . 356

5.3.2.13 Aerosols . . . . . . . . . . . . . . . . . . . . 356

5.3.2.14 Lacquers . . . . . . . . . . . . . . . . . . . . . . . . . . 357

5.3.3 Application Areas of Cosmetic Products . . . . . . . . . . . . 357

5.3.3.1 Skin . . . . . . . . . . . . . . . . . . . . . . . . . . . . 357

5.3.3.2 Appendages of the Skin . . . . . . . . . . . . . . . . . . . 359

Hair . . . . . . . . . . . . . . . . . . . . . . . . . . 359

Sebaceous glands . . . . . . . . . . . . . . . . . . . . . . . . . 360

Small (eccrine) sweat glands . . . . . . . . . . . . . . . . . 360

Large (apocrine) sweat glands . . . . . . . . . . . . . . . 361

Nails . . . . . . . . . . . . . . . . . . . 361

5.3.3.3 Functions of the Skin and its Appendages . . . . . . . . . . . 361

5.3.3.4 Adaptability of the Skin . . . . . . . . . . . . . . . . . . 362

5.3.3.5 Oral Cavity . . . . . . . . . . . . . . . . . . . . . . . . 362

Mucous membrane of the mouth . . . . . . . . . . . . 362

Teeth . . . . . . . . . . . . . . . . . 363

5.3.4 Surfactants in Cosmetic Products . . . . . . . . . . . . . . . 364

5.3.4.1 Interactions of Surfactants with the Skin . . . . . . . . . . . . 364

5.3.4.2 Interactions of Surfactants with the Hair . . . . . . . . . . . . 365

5.3.4.3 Interactions of Surfactants with the Oral Cavity and the Teeth . . 366

5.3.4.4 Predominant Surfactants for Cosmetic Products . . . . . . . . . 366

Anionic surfactants . . . . . . . . . . . . . . . 366 
Cationic surfactants . . . . . . . . . . . . 368

Amphoteric surfactants . . . . . . . . . . . . . . . . . . 368

Nonionic surfactants . . . . . . . . . . . . . . . . . . . 369

5.3.4.5 Evalutation of Surfactants for Cosmetic Products . . . . . . . . 369

5.3.5 Classification of Cosmetic Products . . . . . . . . . . . . . . 370

5.3.5.1 Possibilities for the Classification of Cosmetic Products . . . . . . . 370

5.3.5.2 Cosmetic Products, the Effect of Which is Primarily Assignable to the

Content of Surfactants . . . . . . . . . . . . . . . . . . 371

Skin cleaning preparations . . . . . . . . . . . . . 371

Bath preparations . . . . . . . . . . . . . . . . . . . . 372

Skin care preparations . . . . . . . . . . . . . . . . 373

Skin creams . . . . . . . . . . . . . . . . . . . 373

Body lotions . . . . . . . . . . . . . . . . . . . . . 373

Facial lotions . . . . . . . . . . . . . . . . . . 373

Skin oils . . . . . . . . . . . . . . . . . . . . . . . 373

Hydrophilic skin oils . . . . . . . . . . . . . . . . . . . . 374

Hair care products . . . . . . . . . . . . . . . . . . . . 374

Shampoos . . . . . . . . . . . . . . 374

Hair rinses . . . . . . . . . . . . . . . . . . . . . . 374

Hair conditioners . . . . . . . . . . . . . . . 374

Hair colorants . . . . . . . . . . . . . . . . . . . 374

Shaving preparations . . . . . . . . . . . . . . . . . . . 375

Shaving soaps . . . . . . . . . . . . . . . 375

$\begin{array}{ll}\text { 5.3.5.3 } & \text { Cosmetic Products, in Which Surfactants } \\ \text { Perform Auxiliary Functions . . . . . . . . . . . . . . . . . } 375\end{array}$

Shaving lotions . . . . . . . . . . . . . . . . 375

Hair dressing emulsions . . . . . . . . . . . . . . . . . . 375

Hair setting preparations . . . . . . . . . . . . 375

Mouth and tooth care preparations . . . . . . . . . . . . . 376

Toothpowders . . . . . . . . . . . . . . . 376

Toothcreams and toothpastes . . . . . . . . . . 376

Mouthwash or mouth rinse preparations . . . . . . . . 377

Fragrances, perfumes, colognes . . . . . . . . . . . . . . . 377

5.3.6 Performance Evaluation of Cosmetic Products . . . . . . . . . 377

5.3.6.1 Restrictions Imposed by the Legislator . . . . . . . . . . . . . 378

5.3.6.2 Evaluation of the Effect on the Skin . . . . . . . . . . . . . . 378

5.3.6.3 Evaluation of the Effect on the Hair . . . . . . . . . . . . . . . . . 379

5.3.6.4 Evaluation of the Effect on the Teeth . . . . . . . . . . . . . 379

5.3.7 Evaluation of Product Safety . . . . . . . . . . . . . 380

5.3.8 Evaluation of Stability . . . . . . . . . . . . . . . . 380

5.3.9 Testing for Microbial Purity . . . . . . . . . . . . . . . . 381

5.3.10 Guide Compositions . . . . . . . . . . . . . . . . . . . . 382

$5.4 \quad$ Literature Sections 5.1, 5.2, 5.3 . . . . . . . . . . . . . . . 388

6 Manufacture of Consumer Products

(Dr. W. Adler, G. Thor)

6.1 Manufacture of Powder Products 
6.1.1 Introduction . . . . . . . . . . . . . . . . . . . . 400

6.1.2 Manufacturers of Spray Drying Equipment and Installations . . . 400

6.1.3 Process Principles and Historical Review . . . . . . . . . . . . 400

6.1.4 Pressure Nozzle Atomization . . . . . . . . . . . . . . . . . 402

6.1.4.1 Slurry Preparation and Transport . . . . . . . . . . . . . . . 402

6.1.4.2 Air Flow Pattern . . . . . . . . . . . . . . . . . . . . . . 404

6.1.4.3 Process Regulation and Control . . . . . . . . . . . . . . . . 406

6.1.4.4 Blending of the Products to Obtain the Final Product . . . . . . . 408

6.1.4.5 Chemistry of the Ingredients of Detergents and Cleaning Compounds 408 Surfactants . . . . . . . . . . . . . . . . . 408

Phosphates . . . . . . . . . . . . . . . . . . . . . . 410

Sodium aluminum silicates . . . . . . . . . . . . . . . . 411

6.1.5 Mixing and Spray Mixing Processes . . . . . . . . . . . . . 411

6.2 Manufacture of Liquid Products . . . . . . . . . . . . . . 413

6.2.1 Detergents and Cleaning Compounds . . . . . . . . . . . . 413

6.2.2 Shampoos and Hair Rinses . . . . . . . . . . . . . . . . . . 417

6.3 Manufacture of Medium-Viscosity to High-Viscosity Products . . . 418

6.3.1 Detergents, Cleaning Compounds and Personal Care Preparations

in Paste Form . . . . . . . . . . . . . . . . . . . . . . . 418

6.3.2 Personal Care Preparations in Emulsion Form in the Medium-

to High-Viscosity Range . . . . . . . . . . . . . . . . . . . 419

6.3.3 Mixing Equipment for Batch Processes . . . . . . . . . . . . . 420

6.3.3.1 Mixing Kettles . . . . . . . . . . . . . . . . . . . . . . . 420

6.3.3.2 Trough Mixers and Kneaders . . . . . . . . . . . . . . . 421

6.3.4 Continuous Processes . . . . . . . . . . . . . . . . . . 423

6.3.4.1 One-Shaft Mixers (Extruders) . . . . . . . . . . . . . . . . . 423

6.3.4.2 Two-Shaft Mixers . . . . . . . . . . . . . . . . . . . . . 423

6.3.4.3 Roller Mills . . . . . . . . . . . . . . . . . . . . . . . 425

6.4 Manufacture of Bar Soap from Natural Raw Materials . . . . . . 426

6.4.1 Raw Materials . . . . . . . . . . . . . . . 426

6.4.2 Manufacture of the Basic Neat Soap . . . . . . . . . . . . . . 426

6.4.2.1 Saponification Processes . . . . . . . . . . . . . . . . . . . 426

6.4.2.2 Kettle Saponification . . . . . . . . . . . . . . . . . . . . 427

6.4.2.3 Continuous Saponification Processes . . . . . . . . . . . . . . 427

Neutral fat saponification . . . . . . . . . . . . . . . . . 428

ALFA-LAVAL Centripure Process . . . . . . . . . . . 428

Mazzoni SCN Process . . . . . . . . . . . . . . . . . . 429

Weber and Seeländer KVN Process . . . . . . . . . . . 430

Meccaniche Moderne SAES/N Process . . . . . . . . . . 430

Sharples Process . . . . . . . . . . . . . . . . . . . 430

Monsavon Process . . . . . . . . . . . . . . 431

Fatty acid saponification . . . . . . . . . . . . . . . . . 431

Mazzoni SC and SCC Processes . . . . . . . . . . . . . 431

ALFA-LAVAL High Caustic-High Solids Saponification

Process . . . . . . . . . . . . . . . . . . . . . 431

Meccaniche Moderne SAES/AG Process . . . . . . . . 431

Methylester saponification . . . . . . . . . . . . . 432 
6.4.2.4 Drying Systems . . . . . . . . . . . . . . . . . . 432

6.4.3 Manufacture of Bar Soap . . . . . . . . . . . . . . . . . . 434

Mixing and homogenizing . . . . . . . . . . . . . . 434

Strand formation . . . . . . . . . . . . . . . . . . . 434

Cutting of soap strands into raw bars . . . . . . . . . 435

Stamping . . . . . . . . . . . . . . . . 435

6.4.3.1 Uniformly Colored (Single Color) Bar Soaps . . . . . . . . . . 436

6.4.3.2 Multicolored Bar Soaps . . . . . . . . . . . . . . . . . . . 436

6.4.3.3 Transparent Soaps . . . . . . . . . . . . . . . . . . . . 436

6.4.3.4 Shaving Soaps . . . . . . . . . . . . . . . . . . 436

6.5 Manufacture of Bar Soaps Based on Synthetic Surfactants . . . . 436

6.6 Literature .................. . . 437

7 Analysis of Surfactants and Surfactant Formulations . . . . . . . 440

(Dr. G. Schwarz, Dr. E. Vaeth)

7.1 Introduction . . . . . . . . . . . . . . . 440

7.2 Inorganic Components in Detergents and Cleaning Agents . . . . 440

7.3 Organic Components in Detergents . . . . . . . . . . . . 446

7.3.1 Preface . . . . . . . . . . . . . . . . 446

7.3.2 Raw Material Analysis . . . . . . . . . . . . . . . . 446

7.3.3 Analysis of a Detergent . . . . . . . . . . . . . . . . . . . 447

Nonionic surfactants . . . . . . . . . . . . . . . . . . 448

Anionic surfactants . . . . . . . . . . . . . . . . . . . . 448

Amines and quaternary ammonium compounds (QAC) . . . . 448

Proteases . . . . . . . . . . . . . . . . . . 449

Fluorescent whitening agents. . . . . . . . . . . . . . . . 449

Polycarboxylic acids . . . . . . . . . . . . . . . . . 449

7.3.4 Analysis of Cosmetics and Toiletries . . . . . . . . . . . . . . 449

7.4 Literature . . . . . . . . . . . . . . . . . . . 449

8 Environmental Impact . . . . . . . . . . . . . . . . . . . 450

(Dr. P. Gerike)

8.1 Introduction . . . . . . . . . . . . . . . 450

8.2 Components of Detergents, Cleaners and Cosmetics . . . . . . . 450

8.3 Environmental Aspects. . . . . . . . . . . . . . . . . . 452

8.3.1 Surfactants . . . . . . . . . . . . . . . . 452

8.3.1.1 Methods for Determining Toxicity Towards Water Organisms . . . 453 
8.3.1.2 Toxicity Data. . . . . . . . . . . . . . . . . . 454

8.3.1.3 The "Predicted Environmental Concentration" Concept (PEC) . . 455

8.3.1.4 Biodegradability ...... . . . . . . . . . . . . . 459

8.3.1.5 Biodegradability Test Methods . . . . . . . . . . . . . . . . 459

Biodegradability tests specified in detergent legislation . . . . . 459

Ultimate biodegradability . . . . . . . . . . . . . . 460

Test for detecting recalcitrant metabolites . . . . . . . . . . 463

8.3.1.6 Biodegradability Data . . . . . . . . . . . . . . . . . . . . 464

Data from routine tests . . . . . . . . . . . . . . . . 464

Investigations with radiolabelled surfactants . . . . . . . . 466

8.3.1.7 Environmental Data . . . . . . . . . . . . . . . . 467

8.3.2 Phosphate ................... . . 469

8.3.3 Zeolite A . . . . . . . . . . . . . . . . . . . . . 472

8.3.4 Sodium Perborate . . . . . . . . . . . . . . . . 472

8.4 Conclusions .................... . 473

8.5 Literature . . . . . . . . . . . . . . . . 473

9 Biological Effects, Toxicology and Human Safety . . . . . . . . . 475

(Dr. F. Bartnik, Dr. K. Künstler)

9.1 Introduction . . . . . . . . . . . . . . . . 475

9.2 Biochemical Properties of Surfactants . . . . . . . . . . . . 476

9.3 Toxicokinetics of Surfactants . . . . . . . . . . . . 479

9.3.1 Percutaneous Absorption . . . . . . . . . . . . . . . . . . . 479

9.3.2 Intestinal Absorption and Metabolism . . . . . . . . . . . . . 483

$9.4 \quad$ Local Effects . . . . . . . . . . . . . . . . . . . . . . . . 487

9.4.1 Skin Compatibility _. . . . . . . . . . . . . . . . . . . . . 488

9.4.2 Mucous Membrane Compatibility . . . . . . . . . . . . . . . 491

9.4.3 Sensitization . . . . . . . . . . . . . . . . . 492

9.5 Systemic Effects. . . . . . . . . . . . . . . . 495

9.5.1 Acute Toxicity . . . . . . . . . . . . . . . . . . . . . 495

9.5.2 Chronic Toxicity . . . . . . . . . . . . . . . . . . . 495

9.5.3 Carcinogenicity . . . . . . . . . . . . . . . . . 496

9.5.4 Mutagenicity . . . . . . . . . . . . . . . . . . 496

9.5.5 Embryotoxicity . . . . . . . . . . . . . . . . 497

9.6 Conclusions on Consumer Safety . . . . . . . . . . . . . . . 497

9.7 Summary . . . . . . . . . . . . . . . . . . 498

9.8 Literature . . . . . . . . . . . . . . . . . . . . . . . 499 
10 Economic Aspects . . . . . . . . . . . . . . . . . . . . 504

(Dr. G. Jakobi, Dr. P. Krings, Dr. E. Schmadel)

10.1 Production Volumes and Application Areas of Surfactants . . . . 504

10.1.1 Regional Distribution of Surfactant Consumption . . . . . . . . 504

10.1.2 Regional Breakdown by Application Areas . . . . . . . . . . . 506

10.1.3 Surfactant Consumption in Respect to the Chemical Structure . . . 507

10.2 Detergents and Cleaning Compounds . . . . . . . . . . . . 507

10.2.1 Surfactants in Detergents and Cleaning Compounds . . . . . . . 507

10.2.2 Other Detergent Ingredients . . . . . . . . . . . . . . . 507

10.2.2.1 Builders (Phosphates, Zeolite A, NTA) . . . . . . . . . . . . . 509

10.2.2.2 Bleaching Agents . . . . . . . . . . . . . . . . . . . 510

10.2.2.3 Enzymes . . . . . . . . . . . . . . . . . . . 512

10.2.3 Dishwashing Detergents and Cleaning Compounds . . . . . . 512

10.3 Personal Care Products . . . . . . . . . . . . . . . 513

10.3.1 Surfactants in Personal Care Products . . . . . . . . . . . . . 515

10.3.1.1 Soaps . . . . . . . . . . . . . . . . . . . . . 515

10.3.1.2 Synthetic Surfactants . . . . . . . . . . . . . . . 515

10.4 Literature . . . . . . . . . . . . . . . 516

11 Development Trends . . . . . . . . . . . . . . . . . . 517

(Dr. P. Berth)

11.1 Preamble. . . . . . . . . . . . . . . . 517

11.2 Consumer Products . . . . . . . . . . . . . . . . . 518

11.2.1 Detergents . . . . . . . . . . . . . . . . 518

11.2.2 Dishwashing Detergents . . . . . . . . . . . . . . . . 520

11.2.3 Household Cleaners . . . . . . . . . . . . . . . . . . . . . 520

11.2.4 Personal Care Preparations . . . . . . . . . . . . . . . . 520

11.3 Ingredients of Consumer Products . . . . . . . . . . . . . . 521

11.3.1 Surfactants . . . . . . . . . . . . . . . . . . . 521

11.3.1.1 Alkylbenzene sulfonates . . . . . . . . . . . . . . . . . . . . . . . . . . 522

11.3.1.2 Fatty Alcohol Sulfates and Fatty Alcohol Ether Sulfates . . . . . 523

11.3.1.3 $\alpha$-Sulfo Fatty Acid Esters . . . . . . . . . . . . . . . . . . . 523

11.3.1.4 Alkane Sulfonates . . . . . . . . . . . . . . . . . . . . . . 524

11.3.1.5 $\alpha$-Olefin Sulfonates . . . . . . . . . . . . . . . . . . . . . 524

11.3.1.6 Soaps . . . . . . . . . . . . . . . . . . . . . . . . . . . 524

11.3.1.7 Nonionic Surfactants . . . . . . . . . . . . . . . . . . . . 524

11.3.1.8 Cationic Surfactants . . . . . . . . . . . . . . . . . . . . . 525

11.3.1.9 Other Surfactants . . . . . . . . . . . . . . . . . . . . . . . 525

11.3.1.10 Summary . . . . . . . . . . . . . . . . 525 
11.3.2 Builders .. . . . . . . . . . . . . . . 527

Phosphates . . . . . . . . . . . . . 527

Zeolite A . . . . . . . . . . . . . . . . . . . . . . . . 527

NTA . . . . . . . . . . . . . . . . . 527

11.3.3 Bleaching Agents . . . . . . . . . . . . . . . . . . 528

Chlorine bleach . . . . . . . . . . . . . . . . . 528

Oxygen bleaching agents . . . . . . . . . . . . 528

11.3.4 Other Ingredients of Consumer Products . . . . . . . . . . . . 528

11.4 Outlook ................... 529

11.5 Literature . . . . . . . . . . . . . . . . . 529

12 Definition of Important Terms . . . . . . . . . . . . . . . . 531 (Dr. G. Jakobi)

12.1 Terms .................... 531

12.2 Literature ................. . . 535

Subject Index . . . . . . . . . . . . . . . . . . . . . . . . . . . . . 537 